\title{
Creación de Alternativas Pedagógicas para el Ejercicio del Derecho a la Educación en Jóvenes y Adultos: El Caso del Plan Fines en la Provincia de Buenos Aires (Argentina)
}

\author{
Pedagogical Alternatives Creation for the Exercise of Right to \\ Education in Young and Adult Population: The Case of Fines \\ Plan in Buenos Aires (Argentina)
}

\begin{abstract}
Universidad de Buenos Aires
El presente trabajo parte de la consideración del Plan de "Finalización de Estudios y Vuelta a la Escuela” (Fines) como una alternativa pedagógica que se diseña desde las esferas estatales con el objetivo de generar mejores condiciones para el ejercicio del Derecho a la Educación en la población joven y adulta de la Argentina. Uno de los objetivos que persigue este artículo es reconstruir la trayectoria y modificaciones normativas que experimentó el Plan Fines en la Provincia de Buenos Aires a lo largo de su proceso de implementación. En articulación, se propone indagar sobre los procesos de participación en torno a las modalidades de implementación en los que se involucraron distintos colectivos populares durante el período 2008-2015. Para ello se analizarán las características diferenciales que asumió el Plan en tres distritos de la Provincia de Buenos Aires; dos de ellos ubicados en el área metropolitana y uno en el interior provincial.
\end{abstract}

Ariadna Abritta *

Descriptores: Derecho a la educación, Programas educativos de adultos, Organización no gubernamental, Participación comunitaria.

The present article starts from the consideration of the Plan of "Completion of Studies and Return to School" (Fines) as a pedagogical alternative that is designed from the state spheres with the objective of generating better conditions for the exercise of Education's Right in the young and adult population of Argentina. One of the objectives pursued by this article is to reconstruct the trajectory and normative changes that Fines's Plan in the Province of Buenos Aires experienced throughout its implementation process. In articulation, it is proposed to investigate the processes of participation around the modalities of implementation in which different popular groups were involved during the period 2008-2015. For this purpose, the differential characteristics assumed by the Plan in three districts of the Province of Buenos Aires will be analyzed; two of them located in the metropolitan area and one in the provincial interior.

Keywords: Right to education, Adult education programmes, Nongovernmental organizations, Community participation.

El siguiente artículo es parte de las discusiones pedagógica sostenidas por el grupo de investigación APPEAL (Alternativas Pedagógicas y Prospectivas Educativas para América Latina) radicado en el Instituto de Investigaciones en Ciencias de la Educación (IICE) de la UBA. Asimismo, recoge referencias empíricas extraídas de las entrevistas realizadas para el trabajo de campo de mi tesis doctoral, para cuya realización se contó con la ayuda económica de una beca de Finalización de Doctorado subvencionada por la Comisión Nacional de Investigaciones Científicas y Técnicas (CONICET) de la República Argentina.

*Contacto: ariadna.abritta@gmail.com 


\section{Introducción}

En todas las sociedades existe una cierta configuración hegemónica que determina las funcionalidades de la educación en correspondencia con las estructuras sociales, económicas, culturales y políticas que caracterizan a dichas sociedades. Asimismo, desde comienzos de la modernidad occidental, estas configuraciones instauraron una determinada visión de la gramática escolar deseable que prefigura nuestras representaciones sobre las características y dinámicas propias del hecho educativo (Dussell, 2006)

En la actualidad la configuración hegemónica, constructora de un proyecto de mundo caracterizado por el capitalismo modernizante y globalizante, genera un sin número de personas víctimas, excluidas y vulneradas en calidad de titulares de derechos. Así una considerable proporción de la población mundial, regional, nacional y local se ve impedida de poder ejercer efectivamente sus derechos, entre los cuales se encuentra el derecho a la educación.

Contra ese sistema hegemónico excluyente y contra este proceso de vulneración de derechos educativos es que emergen las alternativas pedagógicas:

Las categorías alternativas pedagógicas permiten recopilar una variedad importante
de eventos que tienen en común elementos que se distinguen del modelo educativo
dominante. Debemos recalcar que el término "alternativas" no es aquí usado como
sinónimo de "educación popular". Se trata de un corte más grueso que incluye como
categoría más específica y delimitada "educación popular". (Puiggrós, 1990, p. 18)

La recopilación, sistematización y análisis de las distintas alternativas pedagógicas que se han construido en nuestra historia regional, a lo largo y ancho de América Latina, continúan habilitando nuevos marcos de sentido para pensar nuestros sistemas educativos contemporáneos (en cualquiera de sus niveles) y la gramática escolar predominante. En otras palabras:

(...) las alternativas cumplen un papel deconstructivo de la lógica establecida, tienen capacidad de poner en evidencia la lógica de la configuración discursiva establecida, tienen capacidad problematizadora.

En particular, no desde el lugar de una "crítica" o una "negación”, sino por el contrario, la alternativa es del orden de la afirmación, propone una cierta organización de sentido. Ese efecto deconstructivo puede ser analizado con independencia del análisis histórico respecto a las posiciones de fuerza en cada coyuntura e incluso de su capacidad de constituirse en hegemónica en una determinada coordenada temporo-espacial. Da una opción, puedo elegir, "tengo alternativa". (Rodríguez, 2013, p. 27)

Partimos entonces de la consideración del Plan de "Finalización de Estudios y Vuelta a la Escuela" (Fines) como una alternativa pedagógica surgida de la propia política educativa reciente de la Argentina. La misma está destinada a jóvenes y adultos que vieron vulnerados con anterioridad su derecho a la educación, por lo cual el Plan Fines busca generar formas organizativas e intervenciones pedagógicas que promuevan las condiciones para poder ejercer efectivamente dicho derecho. 


\section{Surgimiento y trayectoria del Plan Fines (2008-2015)}

Luego de las numerosas críticas realizadas a la Ley Federal de Educación (LFE) (Ley 24.195 del año 1993) y sus desastrosas consecuencias para el sistema educativo argentino, el año 2006 será el momento de consolidación de una nueva infraestructura legislativa.

Existe una amplia producción académica en torno a los efectos nocivos de las reformas estructurales aplicadas en América Latina durante los años de hegemonía del modelo neoliberal. En el campo particular de la educación argentina estas reformas se caracterizaron por una reducción de las responsabilidades del Estado Nacional en materia educativa, por procesos de descentralización descoordinada de las unidades escolares a las provincias, el desfinanciamiento de los sistemas educativos provinciales, el aumento de las tasas de no terminalidad primaria, así como la precarización de las condiciones laborales de los docentes y el deterioro de la infraestructura escolar, entre otros elementos ${ }^{1}$

A modo de antecedentes de esta nueva infraestructura legislativa que se consolidaría en el 2006 bajo la presidencia de Néstor Kirhcner resulta de interés mencionar a:

- Ley $\mathrm{N}^{0}$ 25.864 (2004); que estableció como obligatorio el "ciclo lectivo anual mínimo de ciento ochenta (180) días efectivos de clase".

- Ley Técnico Profesional $\mathrm{N}^{\circ} 26058$ (2005); que permitió una recuperación progresiva y continua de la educación técnica articulada al modelo productivo propuesto.

- La Ley de Financiamiento Educativo $\mathrm{N}^{\circ} 26.075$ (2005); que garantiza un incremento progresivo de la inversión del producto bruto interno en el sector educativo por parte del Estado Nacional y los estados provinciales. Hacia el año 2010 dicho aumento llevó a una inversión del 6\% del PBI en el área de educación.

- Ley de Educación Sexual Integral No 26150 (2006), que crea el Programa Nacional de Educación Sexual en el marco del cual se aprobaron luego los lineamientos Curriculares sobre esta relevante temática.

En el año 2006 se sancionará la Ley Nacional de Educación (LEN) No 26.606 la cual, entre otras modificaciones, establecerá la extensión de la educación obligatoria hasta el nivel medio incluido y re-jerarquizará a la educación de jóvenes y adultos/as que dejará de ser considerada un "régimen especial" para volver a ser una modalidad educativa.

La Ley Federal de Educación de 1993 establecía como "regímenes especiales" a la Educación Especial, la Educación de Adultos y la Educación Artística. Por su parte la LEN establece como modalidades del sistema educativo argentino a la Educación Técnico Profesional, la Educación Artística, la Educación Especial, la Educación Permanente de Jóvenes y Adultos, la Educación Rural, la Educación Intercultural Bilingüe, la Educación Domiciliaria y Hospitalaria, y la Educación en Contextos de Privación de Libertad. Es en dicho contexto de ampliación de la obligatoriedad educativa y de re-jerarquización de la educación de jóvenes y adultos que el Plan Fines (Finalización de Estudios y Vuelta a la Escuela) se diseñará.

1 Para un estudio más exhaustivo de las características que adquirieron estas reformas en el campo educativo latinoamericano y argentino se sugieren el trabajo de Gentili (1998). 
Este programa socio-educativo surge por resolución del Consejo Federal de Educación (CFE) $\mathrm{N}^{\circ} 66$ del año 2008 y su diseño e implementación se encuadran dentro de las competencias del Ministerio de Educación de la Nación Argentina. Inicialmente, el Plan surge con un plazo de implementación preestablecido, en su carácter de programa temporal "a término" (LEN N ${ }^{\circ}$ 26.206, art. 138), que según su resolución de creación (Res. CFE. 66/08) ocuparía el período 2008-2011. Su objetivo original consistía en acompañar a aquellos jóvenes y adultos que adeudaran materias de los últimos años del secundario en su preparación, evaluación, y acreditación de las mismas

Para ello, el plan contemplaba un sistema de tutorías que se desarrollaban dos veces por semana en escuelas público-estatales y eran llevadas adelante por profesores que se desempeñaban en el nivel medio. La población destinataria que se buscaba incluir estaba formada por aquellos jóvenes y adultos de 18 años y más, que, habiendo cursado regularmente dicho nivel, adeudaran la evaluación final de algunas asignaturas para obtener el certificado de finalización de estudios secundarios.

En el año 2010, mediante la suscripción de convenios específicos entre el Ministerio de Educación y el Ministerio de Desarrollo Social de la Nación, el Plan Fines comenzó a articular su implementación con el Programa "Ingreso Social con Trabajo, Argentina Trabaja" (PRIST-AT). Este programa de desarrollo social promueve la creación de cooperativas de trabajo para la realización de obras de infraestructura de baja complejidad y tareas relativas al saneamiento urbano en las principales ciudades del país, registrándose una alta concentración de cooperativas en la Provincia de Buenos Aires y, especialmente, en su área metropolitana (conurbano bonaerense).

Atento a que la mayoría de los y las receptores del PRIST-AT no habían culminado sus estudios secundarios el Gobierno de la Provincia de Buenos Aires, mediante resolución 3520/10 de la Dirección General de Cultura y Educación (DGCYE), promovió la apertura de sedes y comisiones del plan Fines en instituciones y organizaciones sociales inscriptas en los territorios donde los y las cooperativistas desarrollan sus vidas cotidianas, recuperándose así una vieja tradición de la educación de jóvenes y adultos. Esta modalidad se ha caracterizado a lo largo de su historia por la búsqueda de estrategias y emplazamientos que permitan vincular la vida productiva y reproductiva de sus sujetos destinatarios con los dispositivos formativos que se proponen. En este sentido, la proximidad física de los espacios destinados a la formación con los lugares de trabajo o residencia de los jóvenes y adultos ha sido una nota distintiva de la educación de jóvenes y adultos (EDJA) a lo largo de su historia.

Podemos rastrear antecedentes en las disposiciones de la Ley 1420 de 1884 cuando establece en su artículo 11 que se fundarán: "Escuelas para adultos, en los cuarteles, guarniciones, buques de guerra, cárceles, fábricas y otros establecimientos donde pueda encontrarse reunido un número, cuando menos, de 40 adultos ineducados".

Asimismo, y más próximo en el tiempo, podemos identificar esta tradición de la EDJA y su orientación hacia la articulación con organizaciones de la sociedad civil en las experiencias de la Dirección Nacional de Educación de Adultos (DINEA) en el período 73-74 (Rodríguez, 1997) y, más específicamente aún, en la creación de los Centros de Educación Media para Adultos (CENS) mediante la celebración de convenios con organizaciones laborales, sindicales, y barriales (Cabrera, 2006). 
Volviendo al Plan Fines, el mismo adquirirá a partir del 2010, con la articulación con el Ministerio de Desarrollo Social de la Nación, nuevas características que marcarán un cambio decisivo respecto a su diseño original. En primer lugar, el plan Fines ampliará la localización de sus comisiones desde las escuelas públicas secundarias o de la modalidad de adultos iniciales a espacios pertenecientes a organizaciones sociales, ya sea la sede de la cooperativa u otras instituciones con que éstas coordinan para el desarrollo del Programa PRIST-AT (sociedades de fomento, capillas, locales políticos, etc.). En este sentido se recupera una vieja discusión del campo pedagógica que cuestiona la asimilación entre espacio educativo y espacio escolar. La escuela es el dispositivo institucional y organizacional que la modernidad occidental tornó hegemónico para la realización de las tareas de formación y educación. Es la predominante y principal, pero dista de ser la única institución educativa ${ }^{2}$.

Una segunda novedad que se introduce con la articulación con el Ministerio de Desarrollo Social de la Nación consiste en que el Plan Fines ya no restringe su accionar a la preparación de materias adeudadas, sino que se amplía al cursado completo del nivel medio de la modalidad de jóvenes y adultos bajo características específicas. Si bien la intencionalidad inicial del Ministerio de Desarrollo Social, traducida en el diseño del Programa "Argentina Trabaja, enseña y aprende", remitía a promover la terminalidad educativa de los y las cooperativistas mediante una propuesta curricular flexible y cercana a sus ámbitos de desarrollo cotidiano, la implementación concreta dotó a la iniciativa de nuevas características, no planificadas o contempladas en un primer momento. Así, su población receptora se extendió de los y las cooperativistas del Programa PRIST-AT a miembros y vecinos de las comunidades donde éstos se desarrollan.

En términos pedagógico-organizativos, el Plan contempla el cursado total del nivel medio de adultos, con un sistema semipresencial de dos días de cursado semanal, y con una duración total de seis cuatrimestres o tres años, pudiendo dar inicio el ciclo lectivo tanto a comienzos del año como luego del receso de invierno.

En lo relativo a los docentes, los mismos son elegidos generalmente mediante el mecanismo de acto público, y se desempeñan bajo la supervisión de las correspondientes autoridades del sistema educativo (inspectores de adultos de los distintos distritos, inspectores distritales e inspectores regionales, articuladamente con talleristas designados por el Ministerio de Desarrollo Social de la Nación). La particularidad que presenta la selección docente en la Provincia de Buenos Aires es la conformación de listados específicos, por distrito o por región educativa, de docentes que desean desempeñarse en el plan. Estos además de los certificados que demuestran idoneidad para el cargo deben presentar un proyecto pedagógico relativo a la materia para la que se postulan, en el cual deben tener en consideración los contenidos mínimos fijados para el nivel medio de la modalidad de Adultos por la resolución DGCYE 6321/95.

Asimismo, el Plan Fines contempla una organización curricular modular, es decir, por módulo, materia o asignatura, la cual constituye una propuesta organizativa predominante en las tendencias didácticas actuales para la formación de jóvenes y adultos. Misirilis (2009) sostiene que:

2 Para profundizar sobre este aspecto se recomienda la lectura de Varela y Alvarez Uría (1991). 
el núcleo del problema (de la desigualdad educativa) ha sido abordado desde el análisis crítico del dispositivo escolar, más precisamente de los componentes duros (Trilla 1998, Terigi 2006) que organizan la experiencia escolar. Este análisis ha dado lugar a la búsqueda de formatos flexibles de educación que hasta el momento estaban siendo pensados para la franja de adultos, y que ahora anticipan, en términos de edad, los destinatarios que estarían requiriendo de estas formas para continuar los estudios. Los caminos encontrados para la flexibilización están prácticamente concentrados en dos formas: la reorganización del tiempo presencial que se requiere a los alumnos en el dispositivo escolar y la modificación del contenido del nivel obligatorio por el de formación para el trabajo. (p. 131)

En abril del 2012, mediante Resolución de la DGCYE 444/12, se institucionaliza, en el ámbito de la Provincia de Buenos Aires, la ampliación de los destinatarios del Plan Fines al conjunto de jóvenes y adultos mayores de 18 años que no hayan podido comenzar y/o concluir sus estudios secundarios. Asimismo, en dicho año se sanciona la disposición SEDGEYC 99/12 por la cual se reconocen los trayectos educativos previos de los sujetos por la escuela secundaria, con independencia de la culminación del nivel completo. Finalmente, la creación de nuevos programas sociales, como el PROG.R.ES.AR ${ }^{3}$ y el "Ellas Hacen", que contemplan entre sus líneas de acción la promoción de la terminalidad de los estudios obligatorios, produjo una sustantiva ampliación de la matrícula y la cobertura del Plan Fines.

A partir del análisis de las múltiples resoluciones que lo formalizan e institucionalizan, los discursos públicos asociados, las capacitaciones y el material documental desarrollado, como así también de observaciones de campo, se puede sostener que la propuesta pedagógica del Plan Fines retoma discursivamente conceptualizaciones del campo de la Educación Popular ${ }^{5}$. Sin negar la distribución desigual de saberes y las valoraciones sociales hegemónicas que se asigna a cada uno de ellos, el Plan Fines sostiene en sus múltiples fundamentaciones la valoración de la comunicación dialógica entre los participantes del centro educativo: un diálogo que reconozca al educando desde sus diversas experiencias de vida, y que retome y reconozca los saberes experienciales diferenciales que portan los sujetos.

Dicha comunicación dialógica es la que permitiría, por un lado, la construcción de conocimientos (que implica ir más allá de la idea de la tabula rasa y la transmisión pedagógica tradicional y unilateral), la selección de contenidos que permitan la problematización de la situación social, económica, política y cultural en la que se desenvuelven los educandos, y la construcción de lo común a partir de la articulación integradora de las diferencias y particularidades.

Finalmente, la propuesta organizativa del Plan Fines plantea un salto cualitativo o una intensificación de la coordinación Estado-organizaciones populares al plantear un trabajo articulado entre las agencias estatales, el sistema de instrucción público y las organizaciones sociales/populares con inscripción territorial. En numerosos distritos del Conurbano Bonaerense el Plan Fines avanzó en la constitución de espacios institucionales

\footnotetext{
3 "Programa de Respaldo a Estudiantes Argentinos" (PROG.R.ES.AR), destinado a jóvenes de entre 18 y 24 años que no hayan comenzado o culminado sus estudios y no se encuentren trabajando en el momento de percepción de la Beca/Plan. El Plan FinEs se contempla como uno de los posibles servicios educativos para certificar la realización de estudios que el PROG.R.ES.AR requiere parar la asignación del subsidio asociado.

4. En el 2013 se incluye como línea programática dentro del Programa "Argentina Trabaja" destinado a mujeres sin trabajo registrado, que perciben la AUH.

5 Para más referencias en torno a las problemáticas en torno a la conceptualización de educación popular se recomienda consultar Rodríguez (2013).
} 
informales donde se intenta promover la participación ciudadana en el proceso de deliberación y toma de decisiones relativos a los procesos de definición e implementación de políticas públicas (Avritzer, 2002,).

\section{Diseño, implementación y participación en torno al Plan Fines}

Esta breve descripción del desarrollo del Plan Fines en el período 2008-2015 constituye una referencia empírica de cómo los procesos de definición de política pública no son estáticos, sino que remiten a un "ciclo" que se retroalimenta de manera tal que el diseño se modifica en función de los procesos de implementación y evaluación.

Tamayo Sáez (1997) propone pensar a las políticas públicas en el marco de un ciclo con 5 etapas: el mismo comienza por la definición del problema, continuando por la formulación de las alternativas de solución, la adopción de una alternativa (diseño), la implantación de la alternativa seleccionada (implementación), y finaliza con la evaluación de los resultados obtenidos.

Sin embargo, diseño e implementación no serían dos momentos totalmente separados, concomitantes y progresivos del ciclo de políticas públicas en general y de los programas socio-educativos en particular. El diseño de los programas, aprehensibles en resoluciones y disposiciones normativas, orienta la implementación, sin embargo, la misma puede a su vez ir adoptando características no contempladas inicialmente y forzando la creación de nuevos marcos regulativos. En otras palabras, los procesos de implementación no son el reflejo exclusivo de lo dispuesto en la letra formal de los diseños, sino el producto de las negociaciones, conflictos y acuerdos que se establecen entre los actores involucrados en su gestión y desarrollo.

El Plan Fines constituye en este sentido una experiencia concreta de cómo los procesos de implementación exceden a lo dispuesto en el diseño oficial del programa, ya sea en lo respectivo a sus receptores, sus emplazamientos o sus dinámicas de trabajo, y cómo estas modificaciones conllevan a la generación de nuevas orientaciones normativas que engrosan el diseño de la política pública.

Paralelamente, la implementación de políticas educativas destinadas a los sectores más vulnerables de nuestra población recurre cada vez con mayor frecuencia a la articulación y coordinación con organizaciones sociales de enclave territorial y desarrollo comunitario, cercanas a los destinatarios de los programas y planes a los que se busca interpelar. En este sentido, los actores que negocian, disputan y acuerdan en torno a las características que asumirá la implementación de los programas socioeducativos en general y del Plan Fines en particular no se restringen a funcionarios públicos de las administraciones nacionales y/o provinciales y excede sin dudas a inspectores, docentes y administrativos del sistema educativo. Los sectores populares organizados a través de distinto tipo de colectivos sociales y políticos se han convertido en los últimos años en uno de los actores centrales de la "hechura de la política pública", y de las características concretas que los programas socioeducativos adquieren en el territorio.

Esta característica que supone poner en cuestión la idea de receptores pasivos o "beneficiarios" para pensar en participación y protagonismo de los sectores populares organizados es parte de la cosmovisión simbólica de los nuevos populismos 
latinoamericanos que atravesaron a América Latina durante los primeros años del siglo XXI y que en la Argentina se asocian con los gobiernos kirchneristas que presidieron nuestro país entre el 2003 y el 2015. En este sentido, este tipo de prácticas políticas nos invitan a pensar la movilización social y la participación popular desde enfoques teóricos que superen la división taxativa entre prácticas clientelares, que supondrían pasividad por parte de los receptores de recursos materiales y simbólicos provenientes del Estado, y movimientos sociales, en tanto actores autónomos y claramente diferenciables del Estado y sus políticas públicas (Forni y Castronouvo, 2014).

\section{Modalidades de participación popular en torno a la implementación del Plan Fines en la provincia de Buenos Aires}

La implementación del Plan Fines en la Provincia de Buenos Aires adquirió diversas modalidades de participación popular según el nivel local en cuestión. En principio, se tornan notorias las diferencias entre las dinámicas asumidas en el conurbano bonaerense respecto a aquellas propias del interior provincial.

En el caso del conurbano bonaerense (área metropolitana) cobró centralidad la conformación de comisiones del Plan Fines en espacios físicos pertenecientes a organizaciones populares, generando una fuerte presencia territorial del Plan, y habilitando y/o requiriendo (según la visión del actor consultado) la mediación y participación de dichas organizaciones a través de la figura de los referentes educativos. Dichos referentes, principalmente militantes o activistas de las distintas organizaciones barriales y políticas, participaron en distinto tipo de instancias o espacios institucionales informales de toma de decisiones. Estas instancias o espacios, que reciben heterogéneas denominaciones según los distritos considerados, se caracterizaron por articular en el debate a autoridades educativas (inspectores principalmente), organizaciones populares y, en algunos casos, a sindicatos docentes. Su función remitió, sobre todo en las etapas iniciales de implementación, a la definición conjunta de criterios para la apertura de nuevas sedes del Plan, a las características que asumiría la selección docente, y al reconocimiento de las trayectorias educativas previas (institucionalizadas posteriormente por la disposición 99/12 anteriormente mencionada)

Por el contrario, en el interior de la Provincia prevaleció la implementación del Plan en espacios escolares formales, habilitando la creación de comisiones en espacios organizacionales, pero restringiendo su capacidad de incidencia en los procesos de toma de decisiones, y desempeñándose los referentes educativos principalmente en tareas relativas a la administración del espacio físico y el seguimiento de estudiantes y profesores. A continuación, se presenta una breve sistematización de las características asumidas por el plan Fines en tres distritos de la Provincia de Buenos Aires: las jurisdicciones de San Isidro y Avellaneda (ubicadas en el área metropolitana del Conurbano Bonaerense) y la de Tres Arroyos (en el interior provincial).

En el distrito de San Isidro (zona norte del conurbano bonaerense) los entrevistados resaltaron las dificultades y oportunidades de implementación generadas por el considerable universo de destinatarios potenciales y efectivos del Plan. 
En este marco tanto autoridades del sistema educativo como referentes barriales registraron la necesidad de construir criterios conjuntos sobre las características que debían tener los espacios físicos (sedes) destinados a las comisiones del plan Fines. Los mismos no remitieron finalmente a las preferencias iniciales de ninguno de los dos actores, sino que se constituyeron en el proceso de conflicto, negociación y acuerdo propio de la coordinación entre sistema educativo y organizaciones populares. Así, las sedes de las comisiones del Plan Fines en San Isidro no remiten a una institución escolar tradicional pero tampoco se restringen a "comedores" o "merenderos" de las organizaciones populares. Por el contrario, las sedes del Plan son el resultado de la construcción de un dispositivo específicamente educativo dentro de los espacios físicos con que cuentan las organizaciones, destacándose el emplazamiento de mesas y sillas adecuadas para el proceso de enseñanza- aprendizaje como así también la provisión de pizarrones y bibliotecas dentro de los mismos.

Asimismo, y dado el emplazamiento de las comisiones en barrios carenciados y/o villas de emergencia, los actores involucrados fueron construyendo criterios conjuntos en torno a las características que los docentes debían presentar para poder desempañarse adecuadamente en este programa socio-educativo. En este sentido, y sin descuidar la formación docente específica (valuada altamente en las grillas de puntaje docente) se valoró altamente la formación o desempeño en actividades propias de la educación popular y la experiencia de trabajo docente previa en emplazamientos considerados "de riesgo". La consideración de los estudiantes y de los referentes educativos sobre el desempeño de los docentes también fue un elemento de valoración, sobre todo a medida que el Plan Fines iba tomando fuerza en este distrito, cuya primera comisión data del año 2011.

Es en este marco que se impulsó el proyecto de profesores referentes, los cuales constituyeron vías de más y mejor vinculación y comunicación entre las autoridades del sistema, las organizaciones populares y los estudiantes. Dichos profesores eran seleccionados mediante acuerdos entre los inspectores y los referentes educativos barriales con base en su compromiso con la tarea de enseñanza y su forma de interpelación a los estudiantes. En algunos casos, incluso, algunos de estos "profesores referentes" eran asimismo miembros de las organizaciones populares que contaban con títulos docentes, se desempeñaban como docentes del Plan Fines, y de esa manera profesionalizaban su tarea militante que se desarrollaba con anterioridad en dispositivos educativos autogestivos, creados por las propias organizaciones como son los apoyos escolares y/o los bachilleratos populares.

En el distrito de Avellaneda (zona sur del conurbano bonaerense), los procesos de construcción de acuerdos conjuntos en torno a las características que debían asumir los espacios físicos de las comisiones del Plan se reiteran, pero se registra en los relatos de los actores involucrados una mayor articulación entre las autoridades del sistema y las organizaciones populares en torno a los procesos de inscripción, seguimiento y selección docente. Vale la pena mencionar que en este distrito se identifica con mayor facilidad e intensidad la participación de los "talleristas" o promotores del Plan dependientes del Ministerio de Desarrollo Social de la Nación, cuya presencia en el distrito de San Isidro es marginal y que genera, consecuentemente, mayores tareas y responsabilidades para los funcionarios públicos del área educativa.

Los "talleristas" (como son denominados coloquialmente en los territorios) funcionan como un "puente" entre el sistema educativo, los territorios y sus organizaciones, 
desempeñando tareas varias que incluyen lo administrativo (la recolección de documentación y el armado de legajos, por ejemplo), la visita y seguimiento de las comisiones, y la asignación de las mismas a los estudiantes que soliciten su ingreso o reubicación. Dentro de este distrito cobra especial importancia la conformación de una mesa de trabajo conjunto entre autoridades del sistema, referentes educativos de las organizaciones y representantes de los sindicatos docentes. Entre las tareas asumidas por esta mesa de trabajo se encuentran la confección de los listados docentes y la asignación de los correspondientes puntajes que, como se mencionara anteriormente, no restringen su ponderación a la titulación docente habilitante sino que incluye elementos relativos al desempeño de tareas docentes en programas socioeducativos similares, y asimismo la evaluación y puntuación de un proyecto pedagógico cuyo enfoque, metodología y forma de evaluación se tornan centrales para generar oportunidades de aprendizaje significativo para jóvenes y adultos que han experimentado con anterioridad una vulneración de su derecho a la educación.

En este sentido, la conformación de esta "mesa de trabajo" constituye una instancia de mayor institucionalidad que el proyecto de "profesores referentes" mencionado anteriormente para el caso de San Isidro. Esto es así porque implica la creación informal de un dispositivo institucional de encuentro, debate, construcción de criterios comunes y mutua regulación que no se visibiliza en San Isidro, donde la comunicación de las autoridades educativas es radial, es decir, es bilateral con cada una de las organizaciones, centralizando la información y la capacidad de toma de decisiones en la figura del inspector de adultos.

Finalmente, para el caso del distrito de Tres Arroyos (interior de la Provincia de Buenos Aires) resulta de importancia mencionar que en las localidades rurales y balearias de dicha jurisdicción las comisiones del plan Fines se ubican exclusivamente en espacios escolares, tanto de nivel medio como (principalmente) de nivel primario. Por lo tanto, solamente en la ciudad cabecera del partido encontraremos sedes de las comisiones Fines que funcionan en espacios no escolares, pertenecientes a distinto tipo de organizaciones barriales y políticas.

Asimismo, cabe destacar que los docentes que se desempeñan en el Plan son principalmente profesores que ya se desempeñaban en los CENS (Centro de Educación de Nivel Secundario) de la jurisdicción y que la ponderación y construcción de listados para la selección docente recayó exclusivamente en la inspectora de adultos del distrito, con colaboración de la dirección y/o la secretaria académica del CENS pero sin ningún tipo de participación de las organizaciones populares.

Respecto a las formas de articulación y participación de estas últimas en la implementación del plan, las mismas parecieran restringirse a tareas administrativas y de seguimiento de los mecanismos definidos unilateralmente en otras instancias y por otros actores. Tal vez las propias palabras de los protagonistas nos permitan hacernos una imagen más precisa de lo sucedido a nivel territorial:

En algún momento hubo como falta de referentes, lo que pasaba era que no había, no tenían como demasiado, no entendían cuál era el rol del referente, entonces era como que me decían "llámame si tenés algún problema", "mándame un mensajito cuando estén los chicos", pero el lugar, no quiero usar la palabra rol porque no es el adecuado, pero el puesto de referentes supongamos, que es un puesto de trabajo, no lo tenían como demasiado asimilado, ni tenían tampoco la idea de cómo debería ser, de cuál es el contenido, eran simplemente, más que nada, 
estaban dedicados a lo que era administrativo (...) Generalmente no sucedía demasiado, o sea, no sucedía algo extraordinario que ameritara lo que ellos consideraban que era necesario para aparecer, entonces era simplemente comunicar... nos pedían las planillas a la finalización del cuatrimestre o alguna cuestión muy puntual como fue en un momento hacer el Censo, que eso fue coordinado por los referentes y fue delegado a los docentes, los docentes mismos hicimos el Censo. Pero generalmente no aparecían, se dio la organización así y funciona así todavía. (Entrevista propia a docente del Plan Fines de Tres Arroyos. El destacado es propio)

\section{Reflexiones finales}

A lo largo de este artículo se intentó sistematizar los cambios que sufrió el diseño original del Plan Fines a lo largo de su implementación en la Provincia de Buenos Aires. Así, los propósitos, destinatarios/as y dinámicas de trabajo que justificaron la creación del Plan se fueron progresivamente modificando durante la etapa de implementación. Así, este programa fue cobrando distintos rasgos según la atención que despertó en diferentes carteras ministeriales (especialmente en el Ministerio de Desarrollo Social de la Nación), las líneas de política social con las cuales se articuló, y los actores sociales (individuales y colectivos) que interpeló.

Asimismo, en el mismo se intentó indagar sobre los procesos de participación en torno a la implementación del Plan Fines en los que se involucraron distintos colectivos populares durante el período 2008-2015. En este sentido se intenta aportar una mirada "desde abajo" (Svampa, 2000) a los procesos de implementación de políticas y programas socioeducativos. Estos colectivos sociales, cuyas principales características son su inserción territorial (De Sousa Santos, 2001; Zibechi, 2003) y su extracción popular, establecieron nexos de diálogo, negociación, conflicto y acuerdo con los funcionarios públicos responsables de la gestión de este programa socio-educativo.

Para finalizar resulta pertinente esbozar algunas reflexiones en torno a las modalidades de participación popular en la definición de políticas públicas vistas desde sus organizaciones. A título esquemático podemos indicar que las mismas fueron interpeladas como:

- Consultoras en el momento de diseño de la política: este tipo de modalidad participativa incluye la consulta pública en el momento de problematización de la cuestión social implicada, que se suele materializar en reuniones y encuentros de trabajo, así como también en distintas iniciativas ligadas al relevamiento y las encuestas de opinión. Los procesos de construcción y legitimación de la Ley de Educación Nacional podrían constituir un ejemplo de este tipo de participación.

- Como mediadoras en la implementación: a través de la difusión e instalación a nivel territorial de políticas públicas definidas unidireccionalmente desde el Estado (en alguno de sus niveles), principalmente a través de las figuras de "promotores". Aquí abundan los ejemplos de distinto tipo de programas sociales e incluso socioeducativos, del cual el plan Fines es un ejemplo.

- Como miembros protagónicos del proceso de diseño e implementación de políticas públicas; que implica la tematización de los problemas sociales y educacionales por parte de los mismos destinatarios de los programas 
socioeducativos y la participación en los procesos (constantemente interactuantes) de diseño e implementación de las políticas.

Esta última forma de participación requiere de la creación de espacios institucionales formales de participación, que establezcan reglas y mecanismos para la misma, que no "burocraticen" o rigidicen la participación de los sectores sociales organizados, sino que, por el contrario, establezcan modalidades claras de definición que se perpetúen más allá de los gobiernos de turno.

En síntesis, constituye una novedad auspiciosa la construcción de políticas y programas socioeducativos que promuevan alternativas pedagógicas que problematicen la gramática escolar hegemónica y construyan dispositivos diversos que garanticen condiciones para el ejercicio del derecho a la educación.

Asimismo, y dado que nos encontramos en el campo de la educación de jóvenes y adultos, resulta auspicioso que dichas alternativas pedagógicas tiendan a ampliar la capacidad de definición, participación y protagonismo de los sectores populares y sus diversas formas organizacionales. Se requiere, sin embargo, que los espacios de coordinación y definición de política pública se institucionalicen, es decir se formalicen y legitimen, con vistas a garantizar normas y reglas que regulen y perpetúen la participación popular en los procesos de toma de decisiones.

Lamentablemente, para el caso argentino, la falta de agilidad o voluntad política para crear dichos espacios ha generado post 2015 un escenario de incertidumbre no sólo para la continuidad del programa Fines en sí mismo, sino para los espacios de participación e incidencia de los sectores populares organizados en su conjunto.

\section{Referencias}

Avritzer, L. (2002). Democracy and the public space in Latin America. Princeton, NJ: Princeton University Press.

Cabrera, M. E. (2006). El campo de la educación de adultos. Su diversidad conceptual y política. En S. Brusilovsky (Ed.), Educación escolar de adultos (pp. 9-15). Buenos Aires: Ed. Noveduc.

De Sousa Santos, B. (2001). Los nuevos movimientos sociales. Recuperado de http://bibliotecavirtual.clacso.org.ar/ar/libros/osal/osal5/debates.pdf

Dussell, I. (2006). De la primaria a la EGB: ¿Qué cambió en la enseñanza elemental en los últimos años? En F. Terigi (Comp.), Diez miradas sobre la enseñanza primaria (pp. 85-130). Buenos Aires: Siglo XXI.

Forni, P. y Castronouvo, L. (2014). Ni piqueteros ni punteros: Organizaciones populares durante el kirchnerismo. La Plata: EDULP.

Gentili, P. (1998). El consenso de Washington y la crisis de la educación en América Latina. En F. Álvarez Uria (Comp.), Neoliberalismo versus democracia. Madrid: La Piqueta.

Misirilis, G. (2009). Deudas y desafíos en la educación de jóvenes y adultos: Una mirada desde un enfoque político-pedagógico. Recuperado de http://www.unsam.edu.ar/escuelas/humanidades/

Puiggrós, A. (1990). Sujetos, disciplina y curriculum en los orígenes del sistema educativo argentino. Buenos Aires: Galerna. 
Rodríguez, L. (1997). Pedagogía de la liberación y educación de adultos. En A. Puiggrós, Dictaduras y utopías en la historia reciente de la educación argentina (1955-1983) (pp. 289-319). Buenos Aires: Galerna.

Rodríguez, L. (2013). Educación popular en la historia reciente en Argentina y America Latina. Aportes para balance y prospectiva. Buenos Aires: APPEAL.

Svampa, M. (2000). Desde abajo. La transformación de las identidades sociales. Buenos Aires: Biblos.

Tamayo Sáez, M. (1997). El análisis de las políticas públicas. En R. Bañón y E. Carrillo (Comps.), La nueva administración pública (pp. 281-312). Madrid: Alianza Universidad.

Varela, J. y Alvarez Uría, F. (1991). La maquinaria escolar. Madrid: Ed. de la Piqueta.

Zibechi, R. (2003). Los movimientos sociales latinoamericanos: Tendencias y desafíos. Revista OSAL, 7(21), 221-230.

\section{Breve CV de la autora}

\section{Ariadna Abritta}

Licenciada en Ciencias de la Educación por la Facultad de Filosofía y Letras de la Universidad de Buenos Aires (UBA). Candidata al título de Doctora en Ciencia Política por la Escuela de Política y Gobierno de la Universidad Nacional de San Martín (UNSAM). Jefa de Trabajos Prácticos de la Cátedra de Introducción al Estudio de las Organizaciones (EPyG-UNSAM). ORCID ID: OOOO-0002-8557-9669. Email: ariadna.abritta@gmail.com 